\title{
Diseño y construcción de un sistema de lavado en vivo para los aisladores de transformadores
}

\section{Design and Construction of a Live Insulator Washing System for Transformers}

\author{
Lizama-Cámara Y.A. \\ Facultad de Ingeniería \\ Universidad Veracruzana \\ Correo:yahir_lizama@ieee.org \\ Mendieta-Antúnez J.A. \\ Industrias IEM \\ Tlalnepantla, Estado de México, \\ Correo:amendieta.antunez@gmail.com \\ Blanco-Brisset E. \\ Industrias IEM \\ Tlalnepantla, Estado de México, \\ Correo:unamanu@hotmail.com \\ Olivares-Galván J.C. \\ Universidad Autónoma Metropolitana, Unidad Azcapotzalco \\ Correo:jolivare1999@yahoo.com \\ Escarela-Pérez R. \\ Universidad Autónoma Metropolitana, Unidad Azcapotzalco \\ Correo: r.escarela@ieee.org
}

Información del artículo: recibido: septiembre de 2010, aceptado: abril de 2011

\section{Resumen}

A lo largo de la historia de la industria eléctrica se han desarrollado diferentes métodos de limpieza para evitar las fallas de los aisladores de los transformadores debido a la contaminación. Este artículo describe los principales métodos de limpieza aplicables a los aisladores de transformadores, enfatizando el sistema de lavado en vivo tipo fijo con agua a alta presión, método que fue aplicado para realizar la limpieza de los aisladores en el banco de transformadores de 900 MVA de la central eléctrica "Laguna Verde", ubicada en el estado de Veracruz, en México. Se propone una metodología para la limpieza de los aisladores de transformadores, donde se identifican las principales variables a tomar en cuenta (el nivel de tensión de los transformadores, nivel de contaminación de los aisladores, determinación del tiempo óptimo de lavado, cantidad de precipitación de agua, presión óptima del chorro de agua, máxima conductividad del agua y las velocidades de los vientos) y se dan valores de referencias de éstas. Además se presenta un análisis económico al aplicar un método de este tipo en una subestación eléctrica.

\section{Descriptores}

- métodos de limpieza

- lavado de aisladores

- contaminación

- aisladores

- transformadores

- no energizado

- energizado

- alta presión de agua 


\begin{abstract}
Through the electrical industry history there have been developments of different cleaning methods to avoid the insulators flashovers due to pollution. This paper describes the principal cleaning methods applicable to transformers insulators, emphasizing the high pressure fixed-type live insulator washing method, which was applied for cleaning the insulators of 900 MVA transformer bank of the "Laguna Verde" power plant localized at the state of Veracruz in Mexico. We propose a transformer insulator cleaning methodology, which identifies the main variables to take into account (the voltage level of the transformers, the pollution level of the insulators, determination of the optimal wash time, the amount of water, the optimal pressure of water jet, the maximum conductivity of the water and the wind velocity), reference values are given for these variables. In addition, we present an economic cost analysis when applying a method of this kind in an electric substation.
\end{abstract}

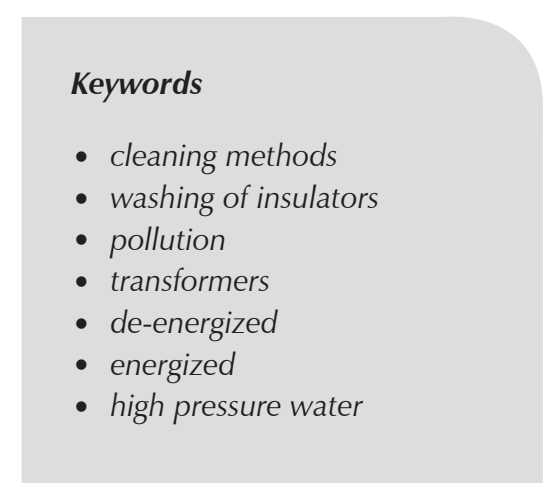

\section{Introducción}

Los transformadores de potencia representan un componente costoso en los sistemas eléctricos de potencia y son de vital importancia en la transmisión de energía eléctrica (Georgilakis et al., 2007). Uno de los principales problemas que se presentan en las centrales eléctricas y en las subestaciones tipo exterior ubicadas en zonas costeras e industriales es que material salino $\mathrm{u}$ otros materiales solubles e insolubles se pueden depositar en la superficie de los aisladores (boquillas) de los transformadores (Cakebread et al., 1978). Si estos materiales no se remueven se forma una capa en la superficie de los aisladores que, al combinarse con la humedad, modifica las características dieléctricas de los mismos, facilitando con esto la producción de arcos eléctricos y cortes en el suministro de la energía, provocando pérdidas económicas debido a fallas en la continuidad del servicio. Investigaciones llevadas a cabo en diferentes países (Suwanasri et al., 2008) muestran que las fallas en los transformadores de potencia, son resultado del envejecimiento (Jongen et al., 2008), y de acuerdo con las estadísticas de ocurrencia, se pueden dividir en:

a) Fallas en el cambiador de derivaciones.

b) Problemas de fugas de aceite dieléctrico en el tanque.

c) Fallas debidas a problemas en los aisladores de los transformadores.

d) Problemas de cortocircuitos en los devanados del transformador.

e) Problemas ocasionados en el núcleo.

f) Otros, como los problemas ocasionados por la temperatura, humedad, etcétera.

En nuestro país, la estadística de ocurrencia de fallas en transformadores de potencia de CFE (Liñan et al., 2001; Guardado et al., 1998), indica que un 26\% de las fallas se deben a problemas en los aisladores de los transformadores (figura 1).

El problema de fallas por contaminación se presenta generalmente en temporadas largas de sequía con alta contaminación ambiental, aunado a la aparición esporádica de neblinas y ligeras lloviznas (Last et al., 1966). Por lo tanto, existe la necesidad de implementar métodos de limpieza (Perin et al., 1995) para eliminar los materiales contaminantes. El tipo de material del aislador, su construcción, si la línea está o no energizada (IEEE std., 1995), son factores importantes para seleccionar el método adecuado; cada método tiene diferentes requerimientos que se deben cumplir para asegurar la correcta limpieza de los aisladores y así evitar problemas que puedan surgir por una mala aplicación.
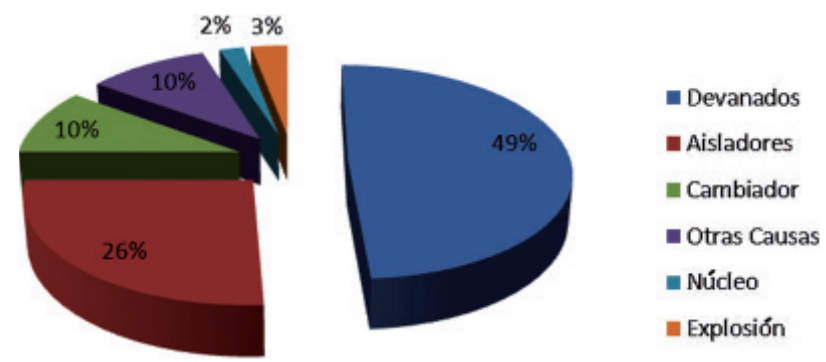

Figura 1. Estadística de fallas en transformadores de potencia 1983-1998 en la red de CFE

Además existen aspectos económicos que se deben considerar al elegir un método de limpieza de aisladores (Last et al., 1966), estos son:

a) El tipo de sistema.

b) La frecuencia de lavado y la cantidad de agua disponible (para sistemas de lavado de aisladores).

c) La localización geográfica de la subestación. 
Los métodos usuales a elegir son: la limpieza a mano, la construcción de un recinto para evitar la contaminación, o con mayores costos, un sistema de lavado en vivo, en el que los costos más importantes corresponden a la bomba, el tanque de almacenamiento y la planta de purificación de agua. También el tamaño de la tubería a utilizar, las válvulas solenoides y el equipo de control aumentan el costo total del método de limpieza.

La tabla 1 muestra los costos de un sistema fijo de lavado en vivo con agua a alta presión. Esta inversión comparada con otros sistemas de limpieza de aisladores, como el sistema tipo móvil (Last, 1966), puede llegar a ser hasta tres veces el valor de dicho sistema; sin embargo, el gasto económico de operación del fijo son hasta dos veces menor que el del móvil, por lo que a largo plazo, la inversión se recuperará.

Tabla 1. Costos del sistema de lavado tipo fijo con agua a alta presión

\begin{tabular}{lc}
\hline \multicolumn{1}{c}{ Equipo a utilizar } & Costo \\
\cline { 2 - 2 } & $\begin{array}{c}\text { Sistema tipo fijo } \\
\text { (En M.N.) }\end{array}$ \\
\hline $\begin{array}{l}\text { Equipo de distribución y almacenaje } \\
\text { (ejemplo: tubería, tanque de almacenaje, } \\
\text { conexiones, válvulas, etc.) }\end{array}$ & 305731 \\
$\begin{array}{l}\text { Equipo adicional (ejemplo: bomba, } \\
\text { purificador de agua, boquillas de lavado, } \\
\text { control, protección, instrumentación, etc.) }\end{array}$ & 609011 \\
$\begin{array}{l}\text { Costo total } \\
\text { Costos de operación }\end{array}$ & 914742 \\
\hline
\end{tabular}

En esta investigación se muestra que el método de limpieza de lavado en vivo tipo fijo con presión alta de agua para aisladores de transformadores, al aplicarlo a una subestación localizada cerca de la costa o en zonas industriales da buenos resultados. Además se muestran varios aspectos del sistema de lavado para la central eléctrica de "Laguna Verde" y se describe una metodología para obtener de forma directa los diseños futuros.

\section{Contaminación de aisladores}

La contaminación que se acumula en la superficie de los aisladores puede estar formada, ya sea por partículas de sal de mar transportadas por el viento en las zonas costeras, o por partículas de cemento, polvo y arena, humos de horno, etcétera que están presentes en las zonas industriales (Hill, 1947). La gran variedad de estas partículas que están en suspensión en el ambiente están descritas y clasificadas por Thompson (1944) y se muestran en la tabla 2.

Tabla 2. Rangos de tamaños de las partículas típicas de contaminación (derechos reservados (C) (Lambeth, 1971), reimpresa con permiso)

\begin{tabular}{ccc}
\hline \multicolumn{2}{c}{$\begin{array}{c}\text { Naturaleza de la materia } \\
\text { en suspensión. }\end{array}$} & Diámetro en $\mu \mathrm{m}$ \\
\hline \multirow{3}{*}{ Inorgánico } & Smog & $0.001-0.3$ \\
& Humo & $0.01-1.0$ \\
& Polvo & $1.0-100.0$ \\
Orgánico & Espocterias & $1.0-10.0$ \\
& Polen & $10.0-20.0$ \\
& Neblina & $15.0-50.0$ \\
Agua & Niebla & $1.0-50.0$ \\
& Llovizna & $100.0-100.0$ \\
& Lluvia & $50.0-400.0$ \\
\hline
\end{tabular}

El grado de contaminación sobre la superficie de los aisladores se puede expresar mediante la densidad del depósito equivalente de sal (ESDD por sus siglas en inglés) en $\mathrm{mg} / \mathrm{cm}^{2}$ (Yamamoto et al., 1961). Este índice nos permite conocer la cantidad de los depósitos en cada aislador, y con base en el mismo, simular las condiciones de operación. Asimismo, mediante pruebas, aplicar los diferentes métodos de limpieza para determinar el más adecuado.

- Para estas pruebas es necesario simular la composición de los depósitos de contaminación alojados en la superficie de los aisladores, esto se logra de la siguiente manera: la materia conductiva de los depósitos de contaminación se simula con cloruro de sodio $(\mathrm{NaCl})$ de acuerdo con el índice ESDD.

- La materia que absorbe la humedad en los depósitos de contaminantes en la superficie de los aisladores se simula con diatomita, ya que este mineral de sílice tiene una capacidad muy alta para absorber líquidos (Shaohua et al., 2009).

- La adhesividad de la contaminación, factor que representa la fuerza necesaria para desprender los depósitos contaminantes de la superficie de los aisladores, se simula mediante la mezcla de un aglutinante (Ruzhang et al., 1993) con la sal y la diatomita.

La concentración máxima permitida del depósito equivalente de sal en la superficie de los aisladores, con la que no se corre riesgo de producir fallas o arqueos es 
$0.03 \mathrm{mg} / \mathrm{cm}^{2}$ (Sakshaug et al., 1982). Con concentraciones del depósito de 0.1 a $0.23 \mathrm{mg} / \mathrm{cm}^{2}$, los aisladores tipo estándar (no del tipo niebla) ya están en riesgo de fallar (Kansong et al., 1980). Con base en estas investigaciones Yamamoto et al. (1961) dan un nivel de contaminación promedio de los aisladores, que se puede utilizar incluso si se trata de tifones que azotan un aislador, dicho valor es $0.05 \mathrm{mg} / \mathrm{cm}^{2}$ para zonas con contaminación promedio y $0.1 \mathrm{mg} / \mathrm{cm}^{2}$ para zonas con severa contaminación.

Existen instrumentos dedicados específicamente para el monitoreo de la contaminación en los aisladores, los cuales miden el pico de corriente de fuga en un periodo dado y el número de pulsos de corriente de fuga que exceden un valor límite dado (Oliviera et al., 2009).

\section{Métodos de limpieza con línea no energizada}

Entre los métodos que se pueden utilizar para realizar la limpieza de los aisladores, los que se realizan con la línea no energizada, y a partir de ahí, trabajadores debidamente capacitados puedan empezar a realizar la labor de limpieza. De estos métodos el más utilizado es el de limpieza a mano, el cual es uno de los más completos y efectivos métodos de limpieza de aisladores, práctico en condiciones de alta contaminación; sin embargo, es muy tedioso, consume una gran cantidad de tiempo y es un proceso sumamente costoso por el simple hecho que el equipo tiene que ser desenergizado.

Al aplicar este método, los trabajadores se tienen que subir en los transformadores para poder llegar a la parte de los aisladores a realizar la limpieza; los trabajadores deben traer consigo lo siguiente: un dispositivo de puesta a tierra personal, fibras finas de acero necesarias para limpiar los aisladores, cinturones de seguridad y una línea de seguridad (IEEE std., 1995).

Aunque este método es necesario para aquellos sistemas que tengan lugares que no se puedan alcanzar con facilidad, como en transformadores, cuya colocación no se haya planeado haciendo imposible el paso de la maquinaria necesaria para el lavado, o donde los componentes a limpiar no pueden soportar altas presiones de agua o aire.

\section{Métodos de limpieza con línea energizada}

Debido a las desventajas que caracterizan a los métodos de limpieza con la línea no energizada, con el tiempo se idearon otros sistemas, éstos ya no necesitaban el corte de energía de la subestación y por lo tanto, su eficiencia era mayor que con los métodos de limpie- za con la línea no energizada. Sin embargo, al tener que trabajar mientras se suministra energía a la red eléctrica, se deben tomar en cuenta precauciones especiales para evitar daños al equipo y al personal que pueda estar en las inmediaciones del transformador. Estos métodos se dividen en aquellos que utilizan aire a presión y los que utilizan agua a distintos niveles de presión. De acuerdo con Yasuda et al. (1976), los métodos con alta presión de agua son los mejores para la limpieza de los aisladores en condiciones de alta contaminación.

Lavado con alta presión de agua, sistema con boquilla en mano

Este método de limpieza es el más utilizado para el lavado de aisladores con agua a presión alta en vivo, este sistema se basa en que trabajadores debidamente calificados pueden efectuar el lavado (Ruzhang et al., 1993), ya sea desde el nivel del piso o mediante el uso de una canastilla elevadora que lo coloca en una posición en la que puede llegar a los aisladores (IEEE std. 1995).

Uno de los parámetros más importantes que se debe tomar en cuenta en este tipo de lavado es la magnitud de la corriente de fuga que pueda pasar desde la parte viva, recorrer el chorro de agua y llegar al trabajador que está operando el sistema. Investigaciones demuestran que se debe limitar dicha corriente de fuga a $1 \mathrm{~mA}$ como valor máximo en las condiciones de tensión máxima de operación. Se debe limitar a $1 \mathrm{~mA}$, ya que este valor es la magnitud máxima de corriente que una persona puede sentir, como ligeras cosquillas. Valores arriba de este nivel (de 2-25mA) pueden llevar a actos involuntarios del cuerpo, como ya no poder soltarse del aparato eléctrico que conduce la corriente y valores aún mayores (de $80-5000 \mathrm{~mA}$ ) pueden ocasionar un paro cardíaco y la muerte (Lee, 1966). De acuerdo con Cawley et al. (2007) entre 1992 y 2002 sucedieron 3,378 incidentes fatales en Estados Unidos, debido a corrientes eléctricas a través del cuerpo de las personas, por lo tanto, es importante limitar la corriente de fuga. Para lograr esto se deben tener en cuenta dos aspectos importantes:

- Las características básicas aisladoras del chorro de agua.

- La distancia mínima que debe existir entre la boquilla de lavado y el aislador.

Ruzhang et al. (1993) encontraron que la corriente de fuga que pasa a través del chorro de agua cambia con el incremento de la distancia que hay entre la boquilla de 
lavado y el aislador energizado, también que dicha corriente se incrementa rápidamente a medida que el diámetro del orificio de la boquilla de lavado se incrementa y que la corriente de fuga se reduce a medida que la resistividad del agua aumenta.

En la figura 2 se muestra la forma en que la corriente de fuga cambia con respecto a la resistividad del agua de lavado, para un diámetro de la boquilla de lavado de $8 \mathrm{~mm}$ a un nivel de tensión eléctrica de $318 \mathrm{kV}$, con una distancia de separación de la boquilla de lavado y la parte energizada de $5 \mathrm{~m}$.

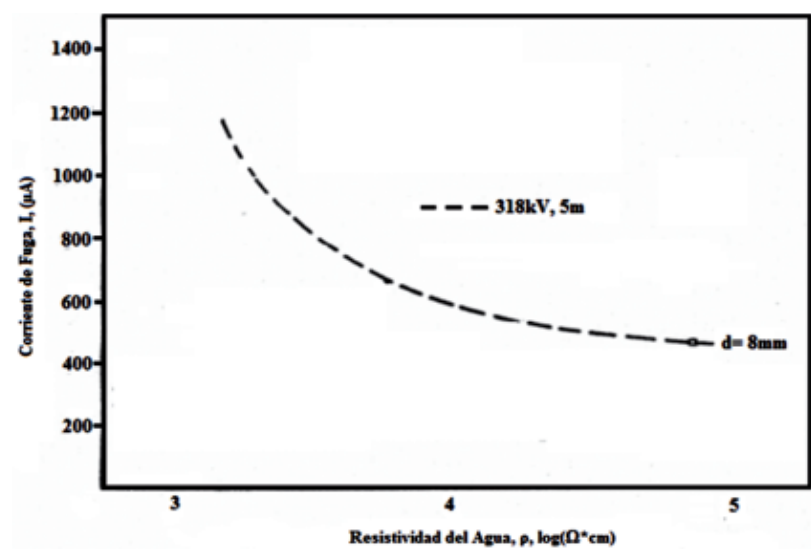

Figura 2. Relación entre la resistividad del agua y la corriente de fuga (derechos reservados (C) (Fujimura et al.,1970), reimpresa con permiso)

\section{Lavado a alta presión de agua, sistema tipo fijo}

El sistema tipo fijo de lavado en vivo con alta presión (SLTFAP) es una medida eficiente contra la contaminación de los aisladores de los transformadores. Sin embargo, este sistema sin las debidas precauciones puede dar origen a arqueos o fallas en el momento en que se está efectuando el lavado de los aisladores, debido a la disminución momentánea de sus propiedades aisladoras mientras son lavados. En la figura 3 se muestra el SLTFAP en operación para la central eléctrica "Laguna Verde", diseñado en esta investigación.

Uno de los requisitos importantes necesario para asegurar el correcto funcionamiento de este método, es el rendimiento de los aisladores mientras los están lavando, para conocer esto se aplica la prueba de resistencia de tensión en el lavado (Fujimura et al., 1970), dicha prueba se describe a continuación: primero, se contamina el aislador a prueba esparciendo sobre el una solución de sal y caolín (Fujitaka et al., 1968). La sal y el caolín representan las sustancias electrolíticas y las partículas de polvo insolubles de la contaminación natural, respectivamente. La tensión de prueba se aplica y se mantiene constante durante todo el ensayo. Entonces se lava el aislador, si la falla o el arqueo ocurre durante el lavado, el mismo procedimiento se vuelve a llevar a cabo otra vez sobre el aislador, volviendo a esparcir la solución de sal y caolín, pero esta vez a $10 \mathrm{kV}$ menos de la tensión de prueba.

Finalmente, la tensión más alta que resistió el aislador cuatro veces consecutivas sin producir arqueo es el que se considera como la resistencia de tensión en el lavado, en otras palabras, es el nivel de tensión máxima que se le puede aplicar al aislador durante el lavado y no se producirá arqueo. Este nivel es muy importante, ya que indica las condiciones en las que el aislador puede fallar.

Otra de las características importantes que se debe tomar en cuenta en este sistema de lavado, es el nivel de contaminación que tienen los aisladores, esto se puede obtener mediante la guía publicada por Yamamoto et al. (1961), para el caso de los aisladores de transformadores, puede ser de hasta $0.10 \mathrm{mg} / \mathrm{cm}^{2}$ en condiciones de alta contaminación.

La cantidad de precipitación de agua es una variable importante, ya que modifica el valor de la resistencia de tensión en el lavado en los aisladores y, por lo tanto, la posibilidad de falla en éstos. La figura 4 mues-

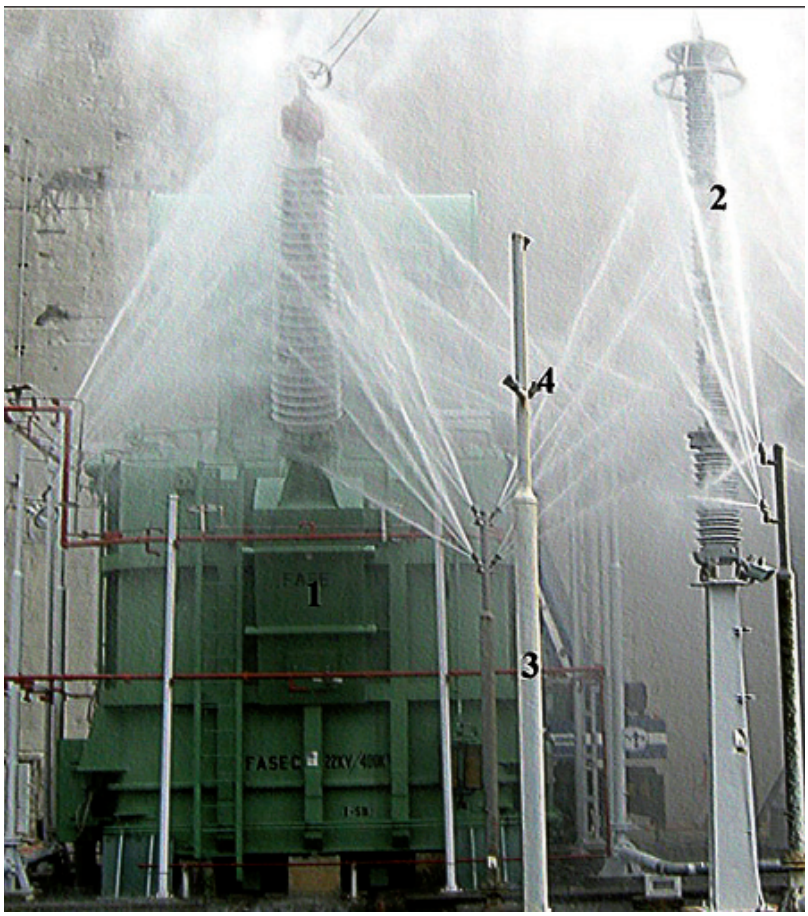

Figura 3. SLTFAP funcionando sobre un transformador monofásico de $300 \mathrm{MVA}$ marca IEM con su pararrayo. 1) transformador marca IEM de 300MVA, 2) pararrayo del transformador, 3) poste de lavado y 4) boquilla de lavado 
tra la relación de la cantidad de precipitación de agua y la resistencia de tensión (Fujimura et al., 1970). Como se puede observar, una baja cantidad de precipitación da como resultado un nivel de resistencia bajo; sin embargo, si la precipitación aumenta, la resistencia se incrementa de la misma forma, pero si la precipitación se eleva aún más, el valor de resistencia disminuye hasta llegar a valores por debajo de $100 \%$, como lo es teniendo una precipitación de más de $25 \mathrm{~mm} / \mathrm{min}$. Por lo tanto, lo ideal es escoger la precipitación que se encuentre sobre la cresta de la curva donde el porcentaje de resistencia de tensión es el más elevado.

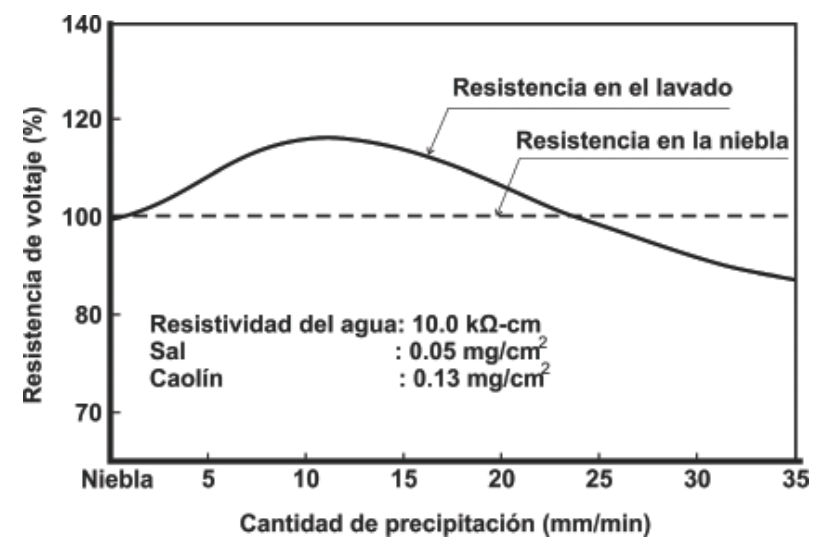

Figura 4. Precipitación y resistencia de tensión (derechos reservados (C) (Fujimura et al., 1970), reimpresa con permiso)

Otra variable a tomar en cuenta en el método de lavado en vivo es el valor de la resistividad que debe tener el agua usada para lavar los aisladores, ya que un bajo nivel de resistividad podría producir arqueos o fallas en los aisladores mientras son lavados. T. Fujimura et al. (1970) demuestran que cuando el agua tiene una resistividad por debajo de $3 \mathrm{k} \Omega-\mathrm{cm}$, el nivel de resistencia de tensión en el lavado empieza a caer significativamente, por lo tanto, el nivel adecuado de resistencia que debe tener el agua que se usa en el sistema de lavado está en el orden de $5 \mathrm{k} \Omega-\mathrm{cm}$, ya que la resistencia de tensión en el lavado tiende a decrecer en la misma forma en que disminuye el valor de la resistencia del agua.

Además, Cakebread et al. (1978) encontraron que la máxima conductividad para el agua de lavado, tiene los siguientes valores típicos: $50 \mu \mathrm{S} / \mathrm{cm}$ (micro Siemens por centímetro) a $400 \mathrm{kV}$, y de $200 \mu \mathrm{S} / \mathrm{cm}$ a un nivel de tensión de $33 \mathrm{kV}$.

Uno de los factores más importantes en este método de limpieza es el tiempo de lavado, ya que marca con qué frecuencia se debe accionar el sistema y esto repercute en el valor económico del mismo. Por lo tanto, se debe calcular un tiempo efectivo para obtener el máximo lavado de los aisladores.

Yasuda et al. (1976) marcan que el tiempo de lavado ideal en el SLTFAP se encuentra en el orden de los 2030 s, para tiempos mayores a 30s el agua usada no tiene utilidad y solamente se desperdicia, también encontraron que la cantidad de agua descargada por la boquilla se incrementa en proporción al cuadrado del orificio de la boquilla y a la raíz cuadrada de la presión de descarga de la misma, esto se cumple hasta una presión de agua de $80 \mathrm{~kg} / \mathrm{cm}^{2}$.

Matemáticamente:

$Q=0.66 C d^{2} \sqrt{p}$

en donde:

$Q$ es el gasto de agua en litros/min,

$C$ es el coeficiente de flujo de la boquilla en $\mathrm{m}^{3} / \mathrm{hr}$,

$d$ es el diámetro de la boquilla en $\mathrm{mm} \mathrm{y}$

$p$ es la presión en $\mathrm{kg} / \mathrm{cm}^{2}$.

Conociendo la cantidad de agua que consumirá el sistema podemos saber las dimensiones del tanque de almacenamiento.

Es necesario usar una cantidad pequeña de agua que impacte con suficiente fuerza en la superficie de los aisladores, capaz de remover los depósitos de contaminación y que además las gotas de agua no brinquen sobre la parte interior de los aisladores, esto para evitar fallas o arqueos. De aquí que la presión de lavado sea también un importante factor a conocer para diseñar un sistema de lavado en vivo de aisladores. Yasuda et al. (1976) realizaron una comparación entre los sistemas que funcionan a $5 \mathrm{~kg} / \mathrm{cm}^{2}$ y los que funcionan a $30 \mathrm{~kg} / \mathrm{cm}^{2}$, obtuvieron que en los sistemas que utilizan la presión mayor, la resistencia de tensión en el lavado se eleva hasta $130 \%$, que el tiempo de lavado es solamente $50 \%$ del requerido por el de menor presión y que la cantidad de agua necesaria es solamente $70 \%$ de la cantidad usada por el de $5 \mathrm{~kg} / \mathrm{cm}^{2}$. Esto hace que el desempeño del sistema se incremente con la presión alta, por lo tanto, se demostró que la presión óptima para el lavado en alta presión de los sistemas fijos es del orden de $30 \mathrm{~kg} / \mathrm{cm}^{2}$, a mayores presiones se necesitarían materiales especiales para que los aisladores sean capaces de soportar esta nueva presión y, por lo tanto, tampoco sería económicamente sostenible.

Otro factor de vital importancia para el correcto funcionamiento del sistema de lavado en vivo es la velocidad del viento, ya que éste puede cambiar la dirección del chorro de agua provocando que no se efectué el la- 
vado correctamente y que haya la posibilidad de falla o arqueo debido al humedecimiento de los depósitos de contaminación a causa del agua de lavado. El rociado de los aisladores solamente es efectivo y seguro hasta velocidades de viento del orden de $7 \mathrm{~m} / \mathrm{s}$ o menos (Kangson et al., 1980). Para velocidades de viento mayores se requiere el uso de pantallas de agua, estas pantallas lanzan un chorro vertical de agua que es llevado por el viento hasta los aisladores, de tal forma que efectúan el lavado; por este motivo las pantallas se colocan sólo en la dirección del viento y son efectivas para velocidades de hasta $20 \mathrm{~m} / \mathrm{s}$ y rachas de viento de $40 \mathrm{~m} / \mathrm{s}$.

La tabla 3 muestra algunos de los parámetros necesarios que deben considerarse cuando se aplica un sistema de lavado energizado (IEEE std, 1995).

En resumen, los factores que deben investigarse para obtener un diseño eficiente del sistema de lavado en vivo tipo fijo con alta presión de agua son los siguientes:

1. Nivel de tensión de la subestación.

2. Tipo de aisladores que se tienen.

3. Efecto de la contaminación sobre los aisladores.

4. Nivel de contaminación de los aisladores.

5. Máxima conductividad que debe tener el agua con relación a la cantidad necesaria para la instalación.

6. Cantidad total de agua tratada a almacenar.

7. Cantidad de precipitación de agua a usar.

8. Determinar la duración óptima de lavado.
9. Velocidades de los vientos en el lugar de la instalación

10. Óptima presión del chorro o del rociado de agua.

11. Control automático de lavado.

\section{Diseño del sistema de lavado de aisladores de la central eléctrica "Laguna Verde"}

La central nucleoeléctrica "Laguna Verde" propiedad de la Comisión Federal de Electricidad, ubicada sobre la costa del Golfo de México, en el km 42.5 de la carretera federal Cardel-Nautla municipio de Alto Lucero, estado de Veracruz en México, cuenta con dos generadores eléctricos de 600MW de la marca Mitsubishi Heavy Industries, con una tensión de salida de $22 \mathrm{kV}$. La central suministra la potencia a la red eléctrica a través de tres líneas de $400 \mathrm{kV}$, dos a la subestación Puebla II, y la tercera a la subestación Poza Rica II, y dos líneas de transmisión más de $230 \mathrm{kV}$ a la subestación de Veracruz II.

Uno de sus transformadores de $400 \mathrm{kV}$, el cual tenía más de 30 años en operación, ya había excedido su vida útil y se cambió por tres transformadores monofásicos nuevos tipo acorazado, clase ONAN/OFAF (enfriamiento con aceite mineral, circulación natural y aire forzado/enfriamiento con aceite mineral, circulación forzada y aire forzado), capacidad de 300MVA cada uno, con una frecuencia eléctrica de trabajo de $60 \mathrm{hz} \mathrm{y}$ sobreelevación de temperatura de $55^{\circ} \mathrm{C}$, voltaje en alta tensión de $400 \mathrm{kV}$ (entre líneas) con conexión en estrella y voltaje en baja tensión de $21.5 \mathrm{kV}$ con conexión delta.

\begin{tabular}{|c|c|c|c|c|}
\hline $\begin{array}{l}\text { Tensión } \\
\text { de línea } \\
(\mathrm{kV})\end{array}$ & $\begin{array}{c}\text { Mínima } \\
\text { distancia } \\
\text { de la boquilla } \\
\text { al aislador }(\mathrm{m})\end{array}$ & $\begin{array}{c}\text { Mínima } \\
\text { resistividad del } \\
\text { agua } \\
(\Omega-\mathrm{cm})\end{array}$ & $\begin{array}{c}\text { Mínima presión } \\
\text { de la boquilla } \\
(\mathrm{kPa})\end{array}$ & $\begin{array}{c}\text { Máximo } \\
\text { diámetro del } \\
\text { orificio de la } \\
\text { boquilla }(\mathrm{mm})\end{array}$ \\
\hline 13 & 1.82 & 1300 & 2758 & 4.76 \\
\hline 13 & 2.74 & 1300 & 2758 & 6.35 \\
\hline 16 & 2.13 & 1300 & 2758 & 4.76 \\
\hline 34.5 & 2.44 & 1300 & 2758 & 6.35 \\
\hline 34.5 & 2.44 & 1300 & 2758 & 4.76 \\
\hline 69 & 2.74 & 1300 & 2758 & 4.76 \\
\hline 69 & 3.66 & 1300 & 2758 & 6.35 \\
\hline 115 & 3.05 & 1300 & 2758 & 4.76 \\
\hline 115 & 3.96 & 1300 & 2758 & 6.35 \\
\hline 230 & 3.66 & 1300 & 2758 & 4.76 \\
\hline 230 & 4.57 & 1300 & 2758 & 6.35 \\
\hline 500 & 6.10 & 3000 & 3792 & 7.94 \\
\hline $400 \mathrm{~cd}$ & 6.10 & 50000 & 5516 & 6.35 \\
\hline
\end{tabular}

Tabla 3. Parámetros para el lavado con línea energizada (derechos reservados (C) (IEEE std., 1995), reimpresa con permiso) 
El transformador "original" tenía su propio sistema de lavado en vivo tipo fijo, que actuaba sobre los aisladores de los transformadores y sus pararrayos, respectivamente; dicho sistema constaba de una serie de seis postes de lavado, divididos de la siguiente manera: dos para el lavado exclusivo de los aisladores, otros dos para el lavado exclusivo del pararrayos y un último para el aislador y para el pararrayo. Además de estos se contaba con un sexto poste para el lavado del neutro del transformador, el cual fue cancelado mediante una brida ciega. Ya que se contaba con la infraestructura del sistema original de lavado, la mejor opción era adaptar este sistema para que funcionara con el nuevo transformador, en vez de optar por aplicar otro método de limpieza, el cual se tendría que construir desde cero. La figura 5 muestra la localización de dichos postes para la nueva disposición del transformador. Todos los postes de lavado están conectados por medio de una tubería que suministra agua desionizada para el lavado.

Con el cambio de transformadores se procedió a rediseñar el sistema original de lavado para ser utilizado sobre los aisladores de los nuevos transformadores y sus pararrayos. Esto se logró de la siguiente manera: se tenía un total de cuatro postes de lavado diseñados para el viejo método de limpieza, sobre estos modelos se rediseñaron los nuevos postes y se llamaron RP-10, RP-11, RP-12 y RP-22, respectivamente. El material de los postes es tubería de acero A-106, y sus propiedades son: densidad de $7860 \mathrm{~kg} / \mathrm{m}^{3}$, esfuerzo de cedencia de 36259.445psi, módulo de Young de 2.901×107 libras/ pulg ${ }^{2}$, relación de Poisson de 0.266 (Megyesy, 1995).

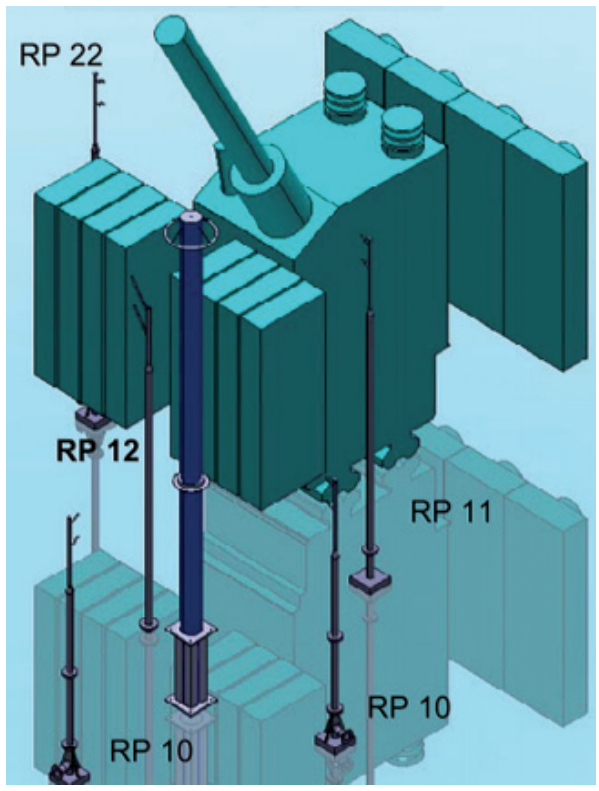

Figura 5. Localización de los postes de lavado
En la figura 6 se muestran los cambios que se realizaron en los postes de lavado RP-11 y se compara con los del sistema original.

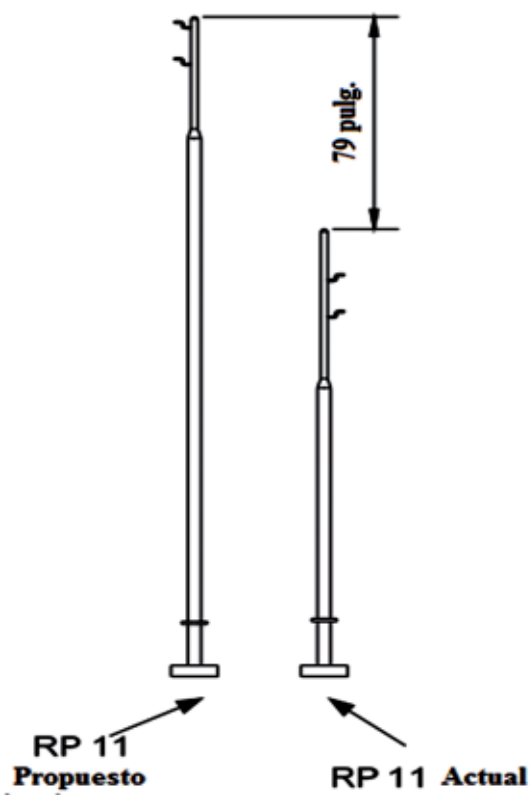

Figura 6. Comparación entre los postes de lavado original y nuevo

El aumento en la longitud de los postes propuestos se basa en la diferencia de alturas de los transformadores, dicha diferencia es la distancia que se aumentó a los postes actuales. Se conserva la distancia entre boquillas de lavado. Todos los postes nuevos se debieron recalcular por el cambio de altura, ya que serían sometidos a fuerzas inerciales provocadas por la probabilidad de la aparición de sismos en la zona, y por el mismo sistema de lavado de aisladores (boquillas de lavado), debido a la alta presión que maneja el sistema $\left(36 \mathrm{~kg} / \mathrm{cm}^{2}\right)$. Matemáticamente,

$F=P A+Q^{2} \frac{\rho}{A}$

en donde:

$F$ es la fuerza de inercia resultante en Newtons,

$P$ la presión del sistema de lavado $\left(36 \mathrm{~kg} / \mathrm{cm}^{2}\right)$,

A el área del orificio de las boquillas de lavado en $\mathrm{cm}^{2}$,

$Q$ el gasto de agua en litros/min y

$\rho$ la densidad del agua en $\mathrm{kg} / \mathrm{m}^{3}$.

La tabla 4 muestra el cálculo de las fuerzas de inercia en cada tipo de poste, en donde A y B se refieren a las boquillas superior e inferior de cada poste de lavado. 
Tabla 4. Cálculo de las fuerzas resultantes del sistema de lavado

\begin{tabular}{lcccc}
\hline $\begin{array}{c}\text { Tipo de } \\
\text { poste } \\
(\mathrm{RP})\end{array}$ & $\begin{array}{c}\text { Presión } \\
\left(\mathrm{kg} / \mathrm{cm}^{2}\right)\end{array}$ & $\begin{array}{c}\text { Área } \\
\left(\mathrm{cm}^{2}\right)\end{array}$ & $\begin{array}{c}\text { Gasto } \\
(1 / \mathrm{min})\end{array}$ & $\begin{array}{c}\text { Fuerza } \\
(\mathrm{N})\end{array}$ \\
\hline $11 \mathrm{~A}$ & 36 & 0.981 & 64 & 358.043 \\
$11 \mathrm{~B}$ & 36 & 0.981 & 56 & 355.324 \\
$12 \mathrm{~A}$ & 36 & 0.981 & 87 & 367.877 \\
$12 \mathrm{~B}$ & 36 & 0.981 & 79 & 364.117 \\
$22 \mathrm{~A}$ & 36 & 0.981 & 74 & 361.950 \\
$22 \mathrm{~B}$ & 36 & 0.981 & 64 & 358.043 \\
\hline
\end{tabular}

La presión interna a la que está sometida el tubo es la presión del sistema $\left(36 \mathrm{~kg} / \mathrm{cm}^{2}\right)$, dicha presión incrementa la rigidez del poste de lavado. Además, también se toman en consideración los posibles sismos y, por lo tanto, se deben considerar en el cálculo de los esfuerzos.

Se tomará como nivel máximo para un sismo que podría suceder en el área lo siguiente: $0.26 \mathrm{~g}$ de aceleración horizontal y $0.19 \mathrm{~g}$ de aceleración vertical, que equivale a un sismo de grado 6.5 en la escala de Richter (Towne et al., 2001), más la fuerza de operación del sistema de lavado.

En este caso, tomamos el poste con mayor fuerza de reacción, si éste es capaz de resistir dichas fuerzas más las creadas por los sismos, los demás postes también serán capaces de soportar y no se presentará la falla del sistema. De acuerdo con la hipótesis de la energía de deformación (Shigley, 2002) en los materiales dúctiles, el poste de lavado se encuentra alejado de la región de falla de fluencia. La figura 7 muestra los esfuerzos máximos de deformación del poste de lavado, debido a las fuerzas de operación de las boquillas, a la presión interna y a las fuerzas debidas a un sismo, de donde se obtuvo que el mayor esfuerzo, al cual está sometido el poste de lavado es de $8810 \mathrm{lb} /$ pulg $^{2}$.

De acuerdo con Megyesy (1995), el esfuerzo máximo permitido de diseño para tubería y accesorios es $17500 \mathrm{lb} / \mathrm{pulg}^{2}$ para tubo A-106 DEC 80. Considerando las posibles fallas que puedan ocurrir en las uniones soldadas, se toma $85 \%$ del esfuerzo máximo de diseño, esto es:

$8810 \mathrm{lb} / \mathrm{pul}^{2}<17500 \mathrm{lb} /$ pulg $^{2 *} 85 \%$

Además, la presión crítica de pandeo para un tubo que está sometido a una presión interna está dada por:

$P c r=\frac{2 \pi^{2} E D t}{(1-2 v) L^{2}}$

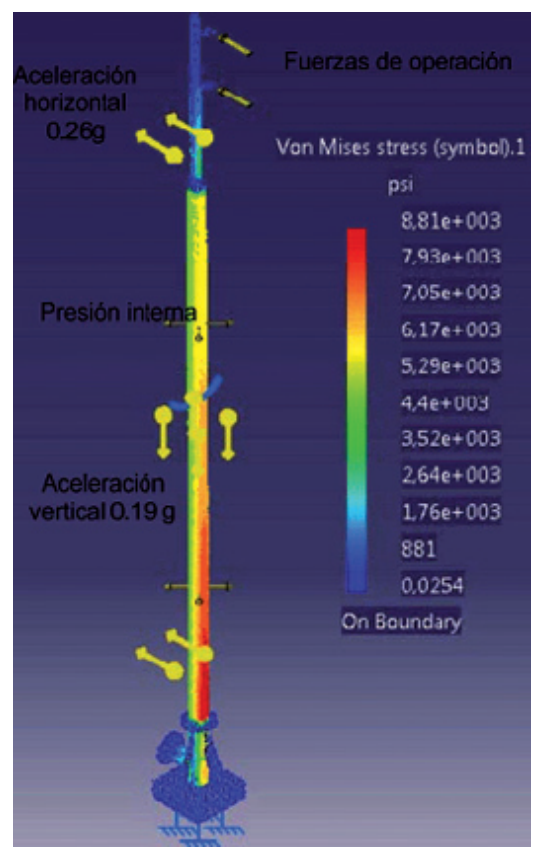

Figura 7. Esfuerzos máximos en el poste de lavado

donde:

Pcr es la presión crítica de pandeo en $\mathrm{kPa}$,

$E$ es el módulo de Young en $\mathrm{Pa}$,

$D$ es el diámetro de la tubería en metros,

$t$ el espesor de la pared en metros,

$L$ la longitud de pandeo en metros y

$v$ es la razón de Poisson (adimensional).

por lo que la presión crítica de pandeo será igual a:

$\operatorname{Pcr}=\frac{2 \pi^{2} * 200 * 10^{9} * 0.127 * 0.0065532}{(1-2(0.28)) *(2(7.406))^{2}}$

$P c r=34,035 \mathrm{kPa}$

Como la presión que maneja el sistema de lavado es de $3,530.4 \mathrm{kPa}$, concluimos que el diseño de los postes de lavado es satisfactorio para los esfuerzos máximos a los que serán sometidos.

\section{Conclusiones}

De los diferentes métodos de limpieza de aisladores, el de lavado a mano es el históricamente más usado en condiciones de línea no energizada; sin embargo, consume una gran cantidad de recursos económicos. Por esta razón los métodos de limpieza con línea energizada o "en vivo" se prefieren, ya que limpian los aislado- 
res de transformadores de forma eficiente, sin embargo, se deben tomar medidas que aseguren el correcto funcionamiento del sistema para evitar posibles fallas que puedan dañar los equipos y a las personas que trabajan en las cercanías de los mismos. Un sistema de este tipo se rediseñó y fue implementado en la subestación eléctrica "Laguna Verde". Debido a que se tenía disponible la infraestructura del sistema original de lavado, la mejor opción era rediseñar dicho sistema para que funcionara apropiadamente con los nuevos transformadores instalados, y no que se instalara un nuevo sistema desde cero o se optara por otro método de limpieza. Para el diseño y construcción de este nuevo sistema de lavado se tomaron en cuenta todas las propiedades eléctricas y mecánicas para poder asegurar que el diseño fuera funcional y se logró el objetivo con excelentes resultados, además, gracias a la experiencia obtenida se pueden diseñar estos tipos de sistemas para otros transformadores. En el diseño del sistema de lavado en vivo tipo fijo con presión alta de agua, que se encuentra operando exitosamente en "Laguna Verde", se consideraron los siguientes factores: nivel de tensión de la subestación, tipo de aisladores que se tienen, nivel de contaminación de los aisladores, máxima conductividad que debe tener el agua con relación a la cantidad necesaria para la instalación, cantidad total de agua tratada a almacenar, cantidad de precipitación de agua a usar, determinar la duración óptima de lavado, velocidades de los vientos en el lugar de la instalación, óptima presión del chorro o del rociado de agua y control automático de lavado.

\section{Referencias}

Cakebread R.J., Brown H.J., Dawkins R.B. Automatic InsulatorWashing System to Prevent Flashover Due to Pollution. Proceedings of the Institution of Electrical Engineers, 125(12): 1363-1366, diciembre 1978.

Cawley J.C., Homce G.T. Trends in Electrical Injury in the US, 1992-2002, en: Petroleum and Chemical Industry Conference $\left(6^{\text {th }}, 2006\right.$, Philadelphia, PA). IEEE Transactions on Industry Applications. Philadelphia, PA. IEEE, 2007, p. 1.

Fujimura T., Okayama M., Isozaki T. Hot-line Washing of Substation Insulators. IEEE Transactions on Power apparatus and Systems. PAS-89 (5): 770-774, mayo 1970.

Fujitaka S., Kawamura T., Tsurumi S., Kondo H., Seta T., Yamamoto M. Japanese Method of Artificial Pollution Test on Insulators. IEEE Transaction on Power Apparatus and Systems, PAS-87(3):729-73, marzo 1968.

Georgilakis P.S., Eleftherios I. Spotlight on Modern Transformer Design. IEEE Power and Energy Magazine, 5(1):40, enero-febrero 2007.
Hill G.L. Tests and Developments in Connection with Hot-Line Insulator Washing. Transactions of the American Institute of Electrical Engineers, 66(1):1203-1216, enero 1947.

Guardado J.L., Olivares J.C., Melgoza E., Venegas V. Modeling of the Non-linear Voltage Distribution in Transformer Windings, en: American Power Conference $\left(60^{\text {th }}, 1998\right.$, Chicago, US). Proceeding of the American Power Conference, pp. 11471152.

IEEE Guide for Cleaning Insulators Standard 957-1995.

Jongen R., Gulski E., Morshuis P., Smith J., Janssen A. Statistical Analysis of Power Transformer Component Life Time Data, en: International Power Engineering Conference $\left(1^{\text {st }}, 2007\right.$, Singapore), IPEC 2007, IEEE, 2008, pp. 1273-1277.

Kansog J.O.C., Mark D. The Laguna Verde 400kV/230kV Hybrid Gas Insulated Substation (HGIS), en: IEEE PES Winter Meeting (1980). IEEE PES Winter Meeting, IEEE, 1980. Paper A-80095-0.

Lambeth P.J. Effect of Pollution on High-Voltage Outdoor Insulators. Proceedings of the Institution of Electrical Engineers, 118(9):1107-1130, septiembre 1971.

Last F.H., Pegg T.H., Sellers N., Stalewski A., Whittaker E.B. Live Washing of H.V. Insulators in Polluted Areas. Proceedings of the Institution of Electrical Engineers, 113(5):847-860. Mayo 1966.

Lee W.R. Death from Electric Shock. Proceedings of the Institution of Electrical Engineers, 113(1):144-148, enero 1966.

Liñan R., Ramírez J., Pascacio A., Nava A. Experiencias en el desarrollo de sistemas de monitoreo y diagnóstico para transformadores de potencia, en: Segundo Congreso Bienal $\left(2{ }^{\mathrm{do}}, 2001\right.$, Irapuato, Guanajuato). Segundo Congreso Bienal, 2001.

Megyesy E.F. Pressure Vessel Handbook, 10rd edition, US: Pressure Vessel Publishing, Inc. 1995, pp. 18-31.

Oliviera S.C., Fontana E., Do Monte de Melo F.J. Real-Time Monitoring of the Leakage Current of $230 \mathrm{kV}$ Glass Type Insulators During Washing. IEEE Transactions on Power Delivery, 24(4): 257-2260, octubre 2009.

Perin D., Pigini A., Visintainer I., Ramamoorty C.M. Live-Line Insulator Washing: Experimental Investigation to Assess Safety and Efficiency Requirements. IEEE Transactions on Power Delivery, 10(1):518-525, enero 1995.

Ruzhang W., Lin S. Safety and Technique of Hot Washing, en: Sixth International Conference on Transmission and Distribution Construction and Live Line Maintenance $\left(6^{\text {th }}, 1993\right.$, Las Vegas, NV.). Proceedings from ESMO-93, IEEE, 1993, pp. 225-239.

Sakshaug E.C., Kresge J.S., Mark D.A., Karady G.G. Contamination \& Hot Wash Performance of Zinc Oxide Station Arresters. IEEE Transactions on Power Apparatus and System,101(5): 1095-1104, mayo 1982.

Shaohua H., Zhipan G., Jing L., Shuibo X. Adsorption of Brilliant Scarlet 3R by Natural Diatomite, en: International Conference on Energy and Environment Technology (2nd, 2009, Guilin, Guangxi). Proceedings from ICEET'09, IEEE, 2009, pp. 397400 . 
Shigley J.E. Mechanical Engineering Design, 8th. Ed., US, Editorial McGraw-Hill, 2002, pp.328-334.

Suwanasri T., Chaidee E., Adsoongnoen C. Failure Statistics and Power Transformer Condition Evaluation by Dissolved Gas Analysis Technique, en: International Conference on Condition Monitoring and Diagnosis $\left(1^{\text {st }}, 2008\right.$, Beijing). Proceedings from CMD 2008, IEEE, 2008, pp. 492-496.

Thompson W.G. The Mechanism of the Contamination of Porcelain Insulators. Journal of the Institution of Electrical Engineers Part II: Power Engineering, 91(22):317-327, agosto 1944.

Towne R.G., Roybal D.D., Ahmed M.A. Earthquake Requirements and Cutler-Hammer Distribution and Control Electrical Equipment Seismic Capabilities. Pittsburg, Pennsylvania: Eaton Corp.SA.125.S.E, mayo 2001.

Yamamoto M., Ohashi K.. Salt Contamination of External Insulation of High-Voltage Apparatus and its Countermeasures. Part III. Transactions of the American Institute of Electrical Engineers Power Apparatus and Systems, 80(3):380-387, abril 1961.

Yasuda M., Fujimura T. A Study and Development of High Water Pressure Hot-Line Insulator Washing Equipment for $500 \mathrm{kV}$ Substation. IEEE Transactions on Power Apparatus and Systems, 95(6):1919-1928, noviembre 1976.

\section{Bibliografía}

Ahmad A.S., Ahmad H., Salam M.A., Tamsir T., Buntat Z., Mustafa M.W. Prediction of Salt Contamination on High Voltage Insulator in Rainy Season Using Regression Technique. Proceedings TENCON 2000, 3(1):184-189, 24-27, septiembre 2000.

Chun S., Park C. Analysis of Contaminants Adhered on Polymeric Insulator Used in Coastal an Industrial Areas, en: Conference on Electrical Insulation and Dielectric Phenomena $\left(1^{\text {st }}, 2008\right.$, Quebec, QC). Annual Report CEIDP, IEEE, 2009, p.1.

Javoronkov M., Abdelaziz C., Fethi G., Zohra B. Prevention of the Interruptions Due the Phenomena of Electric Insulators Pollution, en: First International Symposium on Control, Communications and Signal Processing $\left(1^{\text {st }}, 2004\right)$. First International Symposium on Control, Communications and Signal Processing, IEEE, 2004, pp.493-497.

Vitols A.P., Stead J. Conditioning of Post Insulators and Surge Arresters, en: Electrical Insulation Conference $\left(1^{\text {st }}, 2009\right.$, Montreal, QC). Proceedings from EIC 2009, IEEE, 2009, pp.551554.

\section{Semblanza de los autores}

Yahir Abraham Lizama-Cámara. Técnico en refrigeración industrial pesquera (2006) por el Centro de Estudios Tecnológicos del Mar Núm. 07 del puerto de Veracruz. Actualmente estudia la licenciatura en ingeniería mecánica eléctrica en la Universidad Veracruzana del puerto de Veracruz. Participó en el Verano de la Investigación Científica 2010 haciendo estancia en la UAM-Azcapotzalco y es miembro del IEEE.

Jesús Antonio Mendieta-Antúnez. Es ingeniero mecánico y en sistemas energéticos egresado de la Escuela de Ingeniería de la Universidad La Salle en 2005. Obtuvo el grado de maestro en ciencias en la especialidad de ingeniería eléctrica en el CINVESTAV. Sus áreas de investigación son el control de máquinas eléctricas, sistemas mecánicos vibratorios y control por retroalimentación de estados. De 2009 a la fecha, labora en el área de tecnología de transformadores en industrias IEM SA de CV.

Emmanuel Blanco-Brisset. Estudió ingeniería química con especialidad en tecnología química en la Universidad Iberoamericana. Desde 2003 trabaja en industrias IEM SA de CV en el Departamento de Tecnología y Desarrollo de Transformadores de Potencia como ingeniero en investigación y desarrollo. Sus áreas de interés son modelos de secado por transporte de masa, análisis fisicoquímicos de materiales, sistemas contra incendio y lavado.

Juan Carlos Olivares-Galván. Es ingeniero electricista (1993) y maestro en ciencias (1997) por el Instituto Tecnológico de Morelia. Obtuvo el doctorado en 2004 por el CINVESTAV, unidad Guadalajara. Trabajó por ocho años en la industria como diseñador de transformadores de distribución. En 2004, ingresó como profesor en el Instituto Tecnológico de Zapopan y desde el 2007 es profesor de tiempo completo de la UAM-A. Es miembro del SNI (Nivel I) y Senior Member del IEEE.

Rafael Escarela-Pérez. En 1992 obtuvo el grado de ingeniero electricista de la Universidad Autónoma Metropolitana-Azcapotzalco. En 1993, comenzó sus estudios de posgrado en el Imperial Collage de la Universidad de Londres, donde obtuvo el grado de doctor en 1996. En el mismo año ingresó como profesor de tiempo completo en la Universidad Autónoma Metropolitana. Es miembro del Sistema Nacional de Investigadores (Nivel II) y Senior Member del IEEE. 